\title{
Generating Selected Color using RGB, Auxiliary Lights, and Simplex Search
}

\author{
HyungTae Kim ${ }^{1, a}$, SeungTaek Kim ${ }^{1, b}$, Jongseok Kim ${ }^{1, c}$, KyungChan Jin ${ }^{1, d}$, and Hong-Seok Kim ${ }^{1, e}$ \\ ${ }^{1}$ Smart Manufacturing Technology Group, Korea Institute of Industrial Technology (KITECH), 89 Yangdaegiro-gil, Ipjang-myeon, \\ Seobuk-gu Cheonan 31056, Republic of Korea
}

\begin{abstract}
A mixed light source generates various colors, with the potential to adjust intensities of multiple LEDs, which makes it possible to generate arbitrary colors. Currently, PCs and OSs provide color selection windows that can obtain the RGB or HSL color coordinates of a user's selection. Mixed light sources are usually composed of LEDs in the primary colors, with LEDs in auxiliary colors such as white and yellow used in a few cases. When using auxiliary color LEDs, the number of LED inputs, the dimming levels, is larger than the number of elements in the color coordinate, which causes an under-determined problem. This study proposed how to determine the dimming levels of LEDs based on the selected color. Commercial LEDs have different optical power values and impure color coordinates, even if they are RGB. Hence, the characteristics of the LEDs were described using a linear model derived from the tri-stimulus values (an XYZ color coordinate model) and dimming levels. Color mixing models were derived for the arbitrary number of auxiliary color LEDs. The under-determined problem was solved using a simplex search method without an inverse matrix operation. The proposed method can be applied to a machine vision system and an RGBW light mixer for semiconductor inspection. The dimming levels, obtained using the proposed method were better than derived using other methods.
\end{abstract}

\section{Introduction}

The light sources used in industrial machine vision normally utilize one of the primary colors, red, green, and blue (RGB), whose intensities can be varied by adjusting the input level of the electric current.[1] The light color affects the performance of the inspection machine as well as the circadian rhythms of workers in factories.[2, 3] Hence, the light color is important in manufacturing process but the color selection is limited at the hardware level when using a single light source. A mixed light source can generate various colors by combining RGB LEDs and is constructed using an optical mixer such as a collimator,[4] micro-lens array,[5] shell mixer,[6] diffuser,[7] or bundle of optical fibers.[8] Although such a mixed light source can generate various colors, the spectral ranges of highpower LEDs are constant. Therefore, auxiliary color LEDs are applied to a mixed light source when spectral-range flexibility is required. In this case, the color combination is called 'RGB and white' (RGBW) or 'RGB and yellow' (RGBY). A commercial IC for controlling a four-channel mixed source has already been introduced.[9]

A color mixing algorithm determines the input level, the dimming level, of the mixed light source for a specified purpose using a numerical method. The pur-

\footnotetext{
a e-mail: htkim@kitech.re.kr

be-mail: stkim@kitech.re.kr

ce-mail: jongseok@kitech.re.kr

$\mathrm{d}_{\mathrm{e}-\mathrm{mail}}$ kcjin@kitech.re.kr

e e-mail: hskim@kitech.re.kr
}

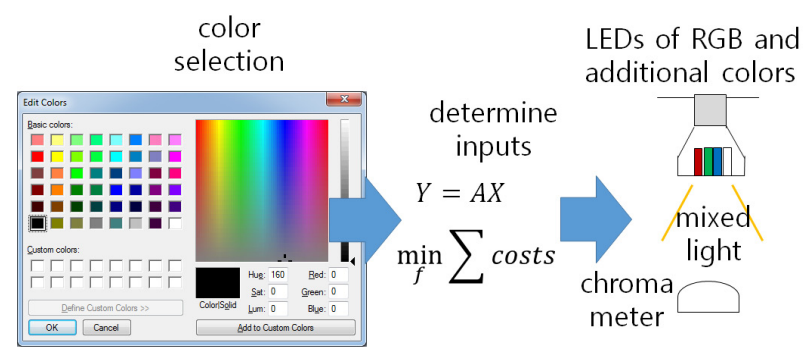

Figure 1. Concept of color selection and light mixing

pose can involve improving the image quality,[10] determining optical spectrum,[11] and aiming desired color coordinate.[12] Commercial LEDs have typical colors. However, these colors usually contain RGB elements even in the case of RGB LEDs. Thus, the color that an LED generates is composed of the primary colors, and a light mixing model can be shown in linear combination with individual LEDs.[13] The linear relation is formed using one of the various color coordinate systems such as tristimulus (XYZ), color temperature and CIE. Linear forms have commonly been applied to RGB mixers, $[14,15]$ and Sisto proposed an expanded model with an arbitrary number of LEDs.[16] Sisto's model can be applied to a mixed light source using auxiliary color LEDs for selected color generation. An RGBW source is the most common type and its linear models were discussed in relation to designing a controller.[15, 17] The color selected by a user has 
Table 1. Coefficients of linear regression

\begin{tabular}{cccccccccc}
\hline \multirow{2}{*}{ LED } & \multicolumn{3}{c}{ X coordinate } & \multicolumn{3}{c}{ Y coordinate } & \multicolumn{3}{c}{ Z coordinate } \\
\cline { 2 - 10 } & $\mathrm{a}$ & $\mathrm{b}$ & $R^{2}$ & $\mathrm{a}$ & $\mathrm{b}$ & $R^{2}$ & $\mathrm{a}$ & $\mathrm{b}$ & $R^{2}$ \\
\hline $\mathrm{R}$ & 1.0001 & 9.3107 & 1.0000 & 0.4274 & 5.7151 & 1.0000 & 0.0007 & -0.0005 & 0.8268 \\
\hline $\mathrm{G}$ & 0.3500 & 51.507 & 0.9987 & 1.4797 & 186.96 & 0.9990 & 0.2309 & -10.306 & 0.9929 \\
\hline $\mathrm{B}$ & 0.5356 & 22.202 & 0.9995 & 0.1450 & 9.3832 & 0.9995 & 3.0871 & 128.65 & 0.9995 \\
\hline $\mathrm{W}$ & 0.7255 & 85.719 & 0.9992 & 0.8223 & 100.97 & 0.9991 & 0.6233 & 56.358 & 0.9994 \\
\hline
\end{tabular}

three elements in the color coordinate system, but the dimming levels for a mixed light source (like RGBW) require additional coordinates. Therefore, there can be many solutions for the dimming level. In this study, a simplex search method (SSM) was used to determine the dimming levels of an RGBW source.

\section{Linear Mixing Model}

\subsection{Additive Color Model for Arbitrary LEDs}

The specific color preferred in the manufacturing field is selected by a human operator. A PC-based system provides a user-friendly interface for selecting this color, such as Windows ${ }^{\mathrm{TM}}$ Paintbrush, as shown in figure 1. The RGB or HSL coordinates can be obtained after selecting the color in the window. The RGB coordinates in a PC are usually called sRGB which can be converted to XYZ valuyse using the following equation.

$$
\left(\begin{array}{c}
X_{s} \\
Y_{s} \\
Z_{s}
\end{array}\right)=c M\left(\begin{array}{l}
R_{s} \\
G_{s} \\
B_{s}
\end{array}\right)
$$

sRGB coordinates have an 8-bit resolution, but the XYZ coordinates can have a higher resolution. In this case, a scale factor $\mathrm{c}$ must be considered. The $\mathrm{M}$ matrix has constant elements which can be found in many articles.[18]

$$
M=\left[\begin{array}{lll}
0.5767 & 0.1856 & 0.1882 \\
0.2974 & 0.6273 & 0.0753 \\
0.0270 & 0.0707 & 0.9911
\end{array}\right]
$$

An actual LED generates a specific color of light. However, this color has all of the RGB elements even if it uses primary color LEDs. In the XYZ color coordinate system, the intensity commonly shows linearity between the dimming levels and generated light color. Generalized coordinate $\mathrm{P}$ in the $\mathrm{XYZ}$ coordinate system and $\mathrm{Q}$ for the dimming level are defined to show this linearity. The following equation is an example for a red LED.

$$
\left(\begin{array}{l}
p_{X} \\
p_{Y} \\
p_{Z}
\end{array}\right)=\left(\begin{array}{l}
a_{R X} q_{R}+b_{R X} \\
a_{R Y} q_{R}+b_{R Y} \\
a_{R Z} q_{R}+b_{R Z}
\end{array}\right)
$$

The general form of the dimming level and XYZ color coordinates can be written as follows.

$$
p_{i}=a_{j i} q_{j}+b_{j i}
$$

where $\mathrm{i}$ is one of the elements of the XYZ color coordinates and $\mathrm{j}$ is one light of multiple LEDs in a mixed light source (-such as one of the RGBWY LEDs). The value of $q_{j}$ can simply be determined as proportional to the RGB space, which is called a direct model. The LEDs in the mixed light source are driven simultaneously using dimming level $q_{j}$ and the XYZ color coordinates of the mixed light, $p_{i}$, can be found as the summation summation of the individual LED lights.

$$
p_{i}=\sum_{j}^{R, G, B, W, \ldots}\left(a_{j i} q_{j}+b_{j i}\right)
$$

Equation (5) can be written in matrix form as follows.[16]

$$
\begin{gathered}
P=Q^{T} A+B \\
A=\left[\begin{array}{lllll}
a_{R X} & a_{G X} & a_{B X} & a_{W X} & \cdots \\
a_{R Y} & a_{G Y} & a_{B Y} & a_{W Y} & \cdots \\
a_{R Z} & a_{G Z} & a_{B Z} & a_{W Z} & \cdots
\end{array}\right] \quad B=\left[\begin{array}{c}
\sum_{j}^{R, G, B, W, \ldots} b_{j X} \\
\sum_{j}^{R, G, B, W, \ldots} b_{j Y} \\
\sum_{j}^{R, G, B, W, \ldots} b_{j Z}
\end{array}\right]
\end{gathered}
$$

A is a not full rank matrix. Therefore, the pseudo-inverse can be applied to obtain Q.

$$
Q^{T}=(P-B)\left(A^{T} A\right)^{-1} A^{T}
$$

Equation (8) is an inverse model and exact solution, which can be physically wrong when it has negative values or is outside the limit. The dimming levels of a light source range from zero to the upper limit. The inverse matrix mathematical operation is sensitive compared to other numerical methods. Therefore optimum methods were considered in this study.

\subsection{Linear Programming}

Linear programming is one of the multi-dimensional, direct, non-differential optimum methods that is used when a problem is composed of linear equations.[19] Therefore, the cost function, constraints, and equations must be written as linear equations. The following equation is a common form of a cost function, and $c^{T}=(1,1,1,1)$ was applied to minimize the dimming levels for the square sum.

$$
\min _{q} f=c^{T} Q
$$

Constraints can be obtained from equation (6) and the nonnegative values of $Q$.

$$
\begin{gathered}
Q^{T} A=P-B \\
q_{j} \geq 0
\end{gathered}
$$

The interior-point method (IPM) was applied to solve this linear programming. 


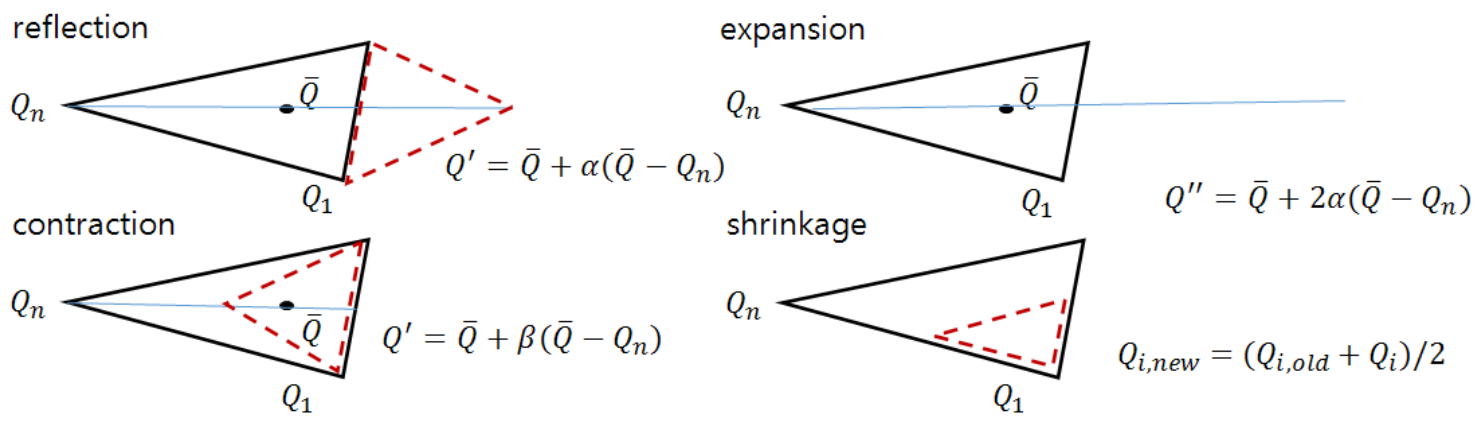

Figure 2. Procedure of simplex search

\subsection{Simplex Search Method}

The SSM is a popular for solving the cost function and constraints. The SSM organizes a probe network using candidate points of dimming inputs, Q. The number of probing points is $\operatorname{dim}(\mathrm{Q})+1$. The best and worst points were defined using an index calculated from the following equation.

$$
\min _{q} f=\sum_{i}^{3}\left(P_{s i}-P_{i}\right)^{2}
$$

The transformation of the simplex in 2D problems is shown in figure 2. An extension line can be defined from the worst point through a centroid. A reflection point can be defined out of the simplex on the extension line. If the reflection point is a new minimum, an expansion point is defined at double the distance of the reflection point on the extension line. The worst point is replaced by one of the reflection and expansion points. If the reflection point is not a new minimum, a contraction point is defined in the simplex on the extension line. The contraction point is not a new minimum, but the simplex is shrunken toward the best point. The sorting of the worst and best points, reflection, expansion, contraction, and shrinkage are iterated until the size of the simplex becomes sufficiently small or does not vary. After determining dimming levels using SSM, the XYZ color coordinate of a mixed light generated by the dimming levels can be converted into CIE1931 xyY coordinates as follows.

$$
(x, y, Y)=\left(\frac{p_{1}}{\sum p}, \frac{p_{2}}{\sum p}, p_{2}\right)
$$

\section{Experiment}

The proposed algorithms were applied to a machine vision system for semiconductor inspection which was presented in our previous studies.[1, 21]. The mixed light source had four high-power LEDs with RGBW colors (KLS-150, Kwangwoo). Its had 11-bit dimming levels, which could be adjusted through RS-232C. The mixed light generated from the color source was transferred through a bundle of optical fibers to a coaxial lens (COAX, Edmund). An industrial camera was attached at the top of the coaxial lens, and the bundle was connected at the side of the coaxial lens. The mixed light was transmitted to a target object in the coaxial lens. The color coordinates of the target object were acquired using a chroma meter (CL-200A, KonicaMinolta). The color selection was made on an RGB coordinate system like the color dialog of Windows Paintbrush. The dimming levels of the mixed light source were determined using the proposed algorithm.

$\mathrm{A}$ and $\mathrm{B}$ matrices had to be obtained for the coefficients of the linear model. XYZ color coordinates were measured using the chroma meter as the dimming level of each LEDs increased. The coefficients and correlation of the measured values were calculated using linear regression. Table 1 lists the coefficients and the correlation between the dimming levels and the color coordinates. Commercial LEDs have XYZ elements, even if they are pure color LEDs, as shown in the table. The correlations were almost 1.0, which indicated good linearity. After the coefficients were determined, the responses of the mixed light source and proposed algorithm were simulated by varying the intensity of the color level in the sRGB space. The RGBW, cyan, magenta and yellow (CMY) intensities in the RGB space increased from zero to the maximum, and the responses were monitored using CIE1931 xyY coordinates.

\section{Results}

The luminance variation by the intensity level of sRGB is shown in figure 3. The targeting line, luminance, was determined by increasing the color intensity, and the solutions obtained using the discussed method must had to followed the black line. The dimming level determined using the SSM most accurately followed the targeting line, compared with the other methods. Figure 4 shows that the CIE 1931 coordinates deviate from the selected color when the blue, yellow and white levels in the sRGB space increased from zero to the maximum. The coordinate variation by the SSM began from the farthest position in figure 4(a), which was the largest deviation for the methods in the experiment. However, it approached zero after the sRGB level increased to $10 / 255$. The coordinate deviations by the IPM and inverse matrix were similar. In figure 4(b) and (c), the coordinate deviations by SSM were the largest in the low luminance. However, they converged to zero after the sRGB level increased to $32 / 255$ and $10 / 255$, which indicates exact color generation. In 

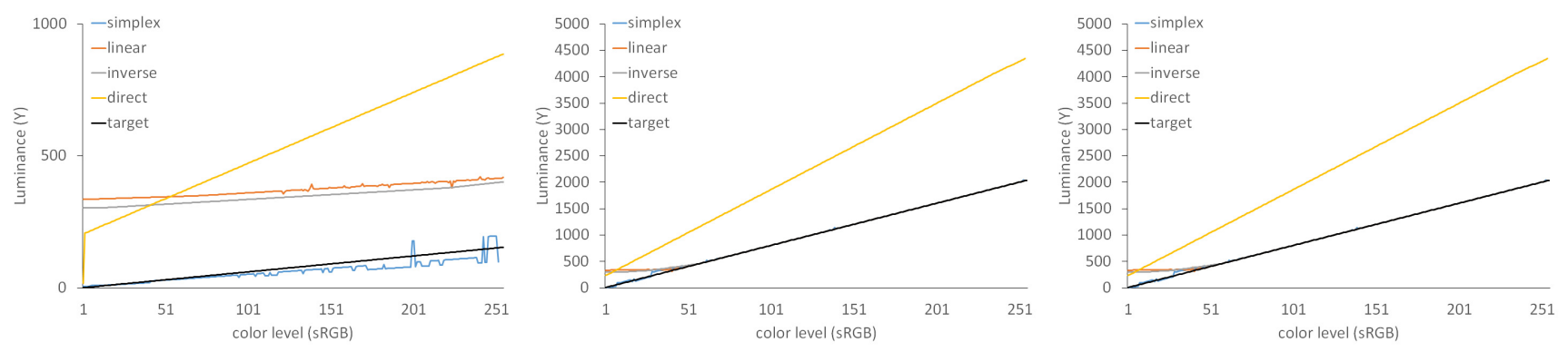

Figure 3. Luminance variation from selected color level (a) blue (b) yellow and (c) white
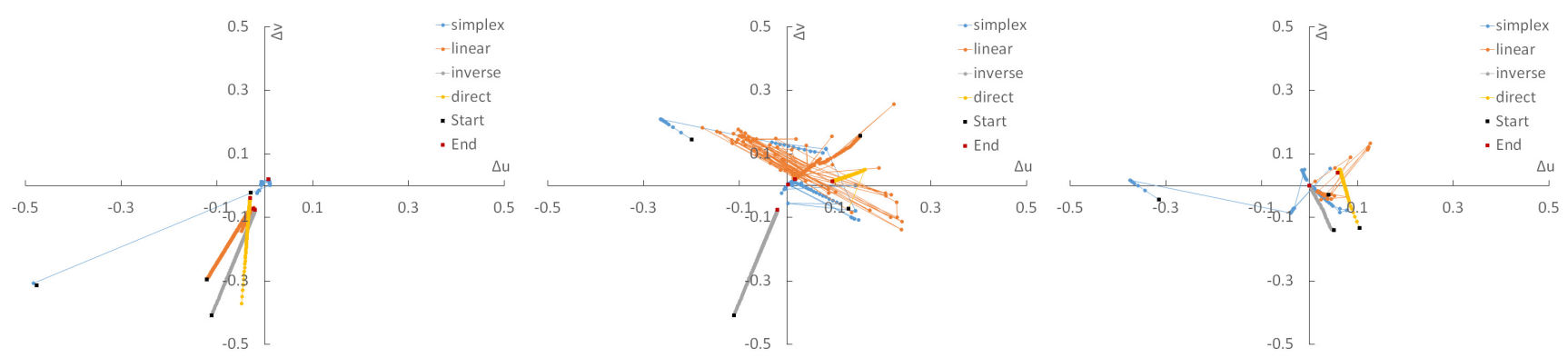

Figure 4. Color coordinates variation (CIE1931 xy) from selected color level (a) blue (b) yellow and (c) white

many cases, the convergence speed by the SSM was faster and the deviation was the smaller under normal luminance compared with the other methods. The SSM showed the lowest deviation except in low dimming levels (approximately 51/255). The characteristics of the color coordinate deviation and luminance were better for an RGBW source. Therefore, the SSM could be used for the accurate generation of selected colors, compared with other methods in normal luminance.

\section{Conclusion}

This study considered the use of the SSM, one of the optimum algorithms, for generating exact colors in industrial lighting systems. Commercial LEDs have all three elements of RGB colors, even if they are called pure color LEDs. Therefore, an exact solution can have an incorrect physical meaning. After constructing a linear model between the dimming levels and XYZ color coordinates, the SSM was used to determine the dimming levels for a selected color. The results show that the proposed method was the most accurate in selected color generation.

\section{References}

[1] H. T. Kim, S. T. Kim, and Y. J. Cho, International Journal of Precision Engineering and Manufacturing 16(2), 247-254 (2015)

[2] S. T. Kim, J. S. Kim, H. T. Kim and Y. K. Kim, Journal of the Optical Society of Korea, 16(4), 449-456 (2012)

[3] H. T. Kim, S. T. Kim, and J. S. kim, International Journal of Optomechatronics, 7(3), 207-221 (2013)
[4] R. P. van Gorkom, M. A.,van AS, G. M. Verbeek, C. G. A. Hoelen, R.G. Alferink, C. A. Mutsaers and H. Cooijmans, Proceeding of SPIE, 6670, 666700E (2007)

[5] J. Muschaweck, Proceeding of SPIE, 7954, 79540A (2011)

[6] J. C. Minano, R. Mohedano, P. benitez and R. Alvarez, SPIE Newsroom, 1-4, Feburary (2013)

[7] H. Wum N. Narendran, Y.Gu and A. Bierman, Proceeding of SPIE, 6669, 666905 (2007)

[8] H. T. Kim, S. T. Kim, Y. J. Cho, International Journal of Optomechatronics, 6(3), 213-225 (2012)

[9] G. Hesse and P. Prendergast, Programmable Analog for High Power LED Color Mixing Applications (Cypress Semiconductor Corp., San Jose, 2007) 1-9

[10] M. H. Lee, D. K. Seo, B. K. Seo and J. I. Park, Optics Letters, 34(17), 2664-2666 (2009)

[11] A. Yano and K. Fujiwara, Plant Methods, 8(11), 46 (2012)

[12] S. Muthu, F. J. Schuurmans and M. D. Pashley, Proceeding of Industry Applications Conference, 1, 327333 (2002)

[13] X. Qu, S. W. Wong and C. K. Tse, Annual Conference of the IEEE Industrial Electronics Society, 13631368 (2007)

[14] K. R. Shailesh and S. Tanuja, Proceeding of IEEE International Advanced Computing Conference, 201-203 (2009)

[15] H. Kim, J. Liu, H. S. jin and H. J. Kim, IEEE Internatioanl Telecommunication and Energy Conference, 1-5 (2009)

[16] M. M. Sisto and J. Gauvin, Proceeding of SPIE, 9190, 919002 (2014) 
[17] M. Tanaka, T. Horiuchi and S. Tominaga, Proceeding of SPIE, 7866, 78660W (2011)

[18] D. Pascale, A Review of RGB Color Spaces...from $x y Y$ to $R^{\prime} G$ 'B' (The BabelColor Company, Montreal, 2003) 20-21

[19] J. S. Arora, Introduction to Optimum Design (2/e)., (Elsevier Academic Press, London, 2004) 191-194
[20] W. H. Press, S. A. Teulkolsky, W. T. Vetterling and B. P. Flannery, Numerical Recipes in C (2/e)., (Cambridge University Press, Cambridge 1992) pp. 430-443

[21] H. T. Kim, K. Y. Cho, K. C. Jin, J. S. Yoon, and Y. J. Cho, International Journal of Optomechatronics, 8(3), 206-217 (2014) 